\title{
Dynamic Force Spectroscopy of Molecular Adhesion Bonds
}

\author{
Berthold Heymann and Helmut Grubmüller \\ Theoretical Molecular Biophysics Group, Max-Planck-Institute for Biophysical Chemistry, 37070 Göttingen, Germany
} (Received 17 December 1999)

\begin{abstract}
Recent advances in atomic force microscopy, biomembrane force probe experiments, and optical tweezers allow one to measure the response of single molecules to mechanical stress with high precision. Such experiments, due to limited spatial resolution, typically access only one single force value in a continuous force profile that characterizes the molecular response along a reaction coordinate. We develop a theory that allows one to reconstruct force profiles from force spectra obtained from measurements at varying loading rates, without requiring increased resolution. We show that spectra obtained from measurements with different spring constants contain complementary information.
\end{abstract}

PACS numbers: 87.15.La, 82.20.Db, 87.15.By, 87.64.Dz

Single molecule mechanical force probe experiments are a powerful and versatile tool for studying molecular adhesion through the response of single molecules to mechanical stress. Investigated processes include specific binding of ligand-receptor [1,2] and antigen-antibody complexes [3], protein unfolding [4,5], and mechanical properties of single polymer molecules such as DNA [6] or polysaccharides $[7,8]$. These experiments probe a molecular force along a reaction coordinate ("force profile"). Because of limited spatial and temporal resolution, however, typically only the maximum of the force profile can be accessed, e.g., by measuring a rupture or unfolding force $[1,4]$. Thus most details of reaction pathways currently remain unresolved.

Molecular dynamics simulations of these experiments [9-11] provided computed force profiles and atomic models of the particular stress response, from which experimental force values could be predicted [7,12]. Biological macromolecules are of particular interest, as the respective force profiles exhibit a complex pattern of barriers that can be related to interatomic interactions, e.g., the rupture of hydrogen bonds, van der Waals contacts, or water bridges.

Theoretical studies considering simple models of the underlying energy landscape $[10,12-14]$ have suggested that direct experimental access to force profiles should be possible through loading rate-dependent force probe experiments ("dynamic force spectroscopy"). Indeed, recent biomembrane force probe (BFP) experiments [2] resolved three distinct regimes for avidin-biotin unbinding; this analysis was based on the two-level-system (TLS) formalism [14,15], which requires one to assume well-separated regimes and is limited to very soft springs.

In this Letter we present a general theory to reconstruct force profiles from dynamic force spectra. A model-free approach is used, i.e., no specific shape of the energy landscape is assumed. Furthermore, we consider the whole range between experiments which use soft springs, e.g., BFP or optical tweezer experiments, and those employing stiff springs, such as atomic force microscopy (AFM), for which the TLS approach is not applicable.
In typical force probe experiments the molecule under study is connected to a "spring," e.g., a laser trap or an AFM cantilever, often via a flexible polymer linker. Subsequently, that spring is moved away from the molecule with velocity $v$, which determines the loading rate $\partial_{t} F$. During that process the maximum force the molecule can withstand - the yielding force-is recorded through the deformation of the spring.

We first aim at a relation between $v$ and $F_{\text {yield, the }}$ yielding force averaged over a series of single molecule experiments. We assume a given suitable reaction coordinate $x$ that describes the process (e.g., the distance between a ligand and its receptor), and a free energy landscape $U_{0}(x)$ governing the unbinding process [bold line in Fig. 1(a)]. We describe the external force $-\partial_{x} V_{\text {pull }}(x, t)$, which usually acts onto the molecule solely in a positive pulling direction, by a semiharmonic potential (dashed line),

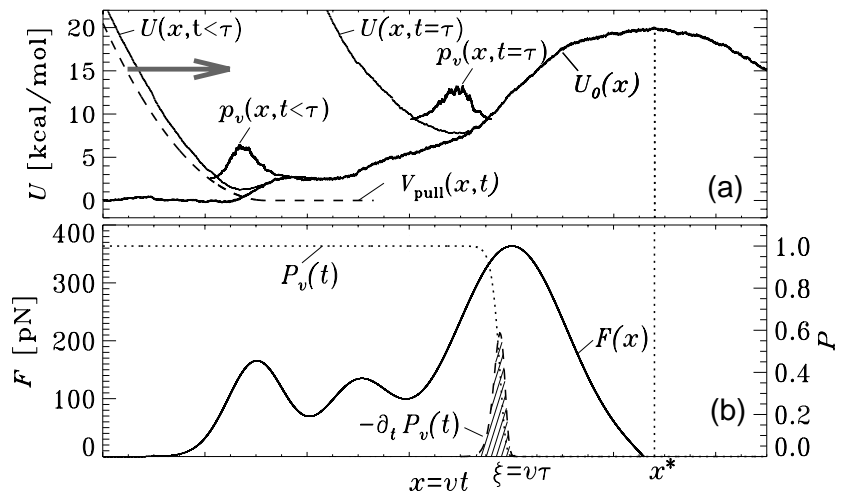

FIG. 1. (a) Single molecule force probe experiments are described by a time-dependent free energy landscape $U(x, t)$ (thin solid lines) along a reaction coordinate $x$, which is shown for two instances, before and at yielding $(\tau)$. $U(x, t)$ involves the free energy landscape $U_{0}(x)$ (bold line) of the unperturbed system, and a semiharmonic potential $V_{\text {pull }}(x, t)$ (dashed line) that describes the external force exerted onto the studied molecule. (b) The fraction $P_{v}(t)$ of bound states (dotted line) and the unbinding flux $-\partial_{t} P_{v}(t)$ (dashed line) define the yielding position $\xi$ and determine which part of the force profile $F(x)=\partial_{x} U_{0}(x)$ (solid line) is probed via $F_{\text {yield }}$ (hatched area). 


$$
V_{\mathrm{pul1}}(x, t)=\left\{\begin{array}{cl}
\frac{1}{2} k(x-v t)^{2} & \text { for } x<v t \\
0 & \text { otherwise }
\end{array}\right.
$$

Here, $k$ is the effective force constant of the spring/linker system. Note that a harmonic potential would suppress activated barrier crossings which, however, are essential for the experiments considered here. As indicated by the gray arrow, the moving spring potential $V_{\text {pull }}(x, t)$ forces the ligand across the barrier. Let $x=0$ be the average reaction coordinate of the unperturbed system, and $V_{\text {pull }}$ is switched on at $t=0$.

For an ensemble of single molecule force probe experiments we assume that the probability distribution $\rho_{v}(x, t)$ of the reaction coordinate, which is governed by picosecond molecular motions, equilibrates rapidly with respect to the experimental milliseconds time scale. In this quasistatic treatment $[10,14,16], \rho_{v}(x, t)$ is given by the Boltzmann factor, $\rho_{v}(x, t)=\exp \left\{-\beta\left[U_{0}(x)+\right.\right.$ $\left.\left.V_{\text {pull }}(x, t)\right]\right\} / Z$, with configurational partition function $Z$ and reciprocal thermal energy $\beta=1 / k_{B} T$. By introducing a partition function $Z_{b}$ of bound states $x<x^{*}$, where $x^{*}$ is the position of the transition state,

$$
Z_{b}=\frac{1}{h} \sqrt{\frac{2 \pi m}{\beta}} \int_{x<x^{*}} d x e^{-\beta\left[U_{0}(x)+V_{\text {pull }}(x, t)\right]},
$$

$\rho_{v}(x, t)=P_{v}(t) p_{v}(x, t)$ can be split into the fraction $P_{v}(t)=Z_{b} / Z$ of bound states and the bound state reaction coordinate distribution $p_{v}(x, t)=\exp \left\{-\beta\left[U_{0}(x)+\right.\right.$ $\left.\left.V_{\text {pull }}(x)\right]\right\} / Z_{b}$, drawn in Fig. 1(a).

For pulling velocities $v>0$ the minimum of $U:=$ $U_{0}+V_{\text {pull }}$ is raised with time as sketched in Fig. 1(a), and the barrier impeding transition is reduced accordingly. Thus, the flux $-\partial_{t} P_{v}(t)$ across the barrier is increased, such that $P_{v}(t)$ drops faster than for $v=0$, and transitions are accelerated according to the Kramers forward rate $k_{\mathrm{TST}}^{+}$,

$$
\partial_{t} P_{v}(t)=-\kappa k_{\mathrm{TST}}^{+} P_{v}(t)=-\omega_{0} P_{v}(t) e^{-\beta \Delta A(v, t)},
$$

where the transmission coefficient $\kappa$ is assumed to be independent of $v ; \omega_{0}:=\kappa /(h \beta)$. The activation free energy is $\Delta A(v, t)=U\left(x^{*}, t\right)-A(v, t)$ with $A(v, t)=-\frac{1}{\beta} \ln Z_{b}$.

If $P_{v}(t)$ were known, and assuming quasistatic conditions, the averaged yielding force could be identified as

$$
F_{\text {yield }}(v)=-\int_{0}^{\infty} d t \partial_{t} P_{v}(t) \max _{t^{\prime}<t} F\left(v, t^{\prime}\right)
$$

with

$$
F(v, t)=\partial_{t} A(v, t) / v .
$$

Since $P_{v}(t)$ is generally sigmoidal [cf. Fig. 1(b)], the mean yielding force can be approximated to sufficient accuracy by $F_{\text {yield }}(v) \approx \max _{t<\tau} F(v, t)$, where the instance of yielding, $\tau$, is defined via $P_{v}(\tau)=\frac{1}{2}$. [Corrections can be derived by expanding $P_{v}(t)$ in $t$ at $\tau$.] The cantilever position at the instance of yielding is $\xi=v \tau$. By using (5) and the time derivative of $Z_{b}$ from (2), a generalized Hooke's law is obtained for harmonic pulling potential,

$$
F(v, t)=k[v t-\langle x\rangle(v, t)],
$$

with average ligand position $\langle x\rangle:=\int x p_{v}(x, t) d x$ [17].

The crucial step is to switch now from $v$ to $\tau$ as the independent parameter, and to approximate $\Delta A(v, t)$ by $\overline{\Delta A}(v, t):=\Delta A(v, \tau)+(t-\tau) \partial_{t} \Delta A(v, \tau)$. This linear expansion in $t$ holds within a yielding period $\tau \pm \Delta t$, for which $|\overline{\Delta A}(v, t)-\Delta A(v, t)|<1 / \beta$. For a compact notation, subsequently the $\tau$ dependency will be omitted, e.g., $A(v, \tau) \equiv A(v)$.

Using $\overline{\Delta A}$ for $\Delta A$ in (3) allows one to solve for $P_{v}(t)$,

$P_{v}(t)=(1-\epsilon) \exp \left[\frac{\omega_{0}}{\beta \partial_{t} \Delta A(v)} e^{-\beta\left[\Delta A(v)+(t-\tau) \partial_{t} \Delta A(v)\right]}\right]$

with $\epsilon \ll 1$ chosen such that $P_{v}(0)=1$ [18].

Recalling the definition of $\tau, P_{v}(\tau)=\frac{1}{2}$, Eq. (7) specifies the activation free energy at the instance $\tau$ of yielding,

$$
\beta \Delta A(v)=-\ln \frac{-\beta \partial_{t} \Delta A(v) \ln 2}{\omega_{0}} .
$$

For later reference note that from (5) it follows that

$$
d A(v) / d v=F(v) d \xi / d v .
$$

Subsequently, we will treat stiff and soft springs separately. Recalling (1), for the stiff pulling potential $V_{\text {pull }}$ shown in Fig. $1(k=1 \mathrm{~N} / \mathrm{m})$, yielding occurs before the maximum $U\left(x^{*}\right)$ is affected by $V_{\text {pull }}$, i.e., $\tau<x^{*} / v$. In this "stiff spring regime" $\left.\partial_{t} U\left(x^{*}, t\right)\right|_{t=\tau}$ vanishes, hence $\partial_{t} \Delta A(v)=-\partial_{t} A(v)=-v F(v)$, and (8) yields

$$
\beta\left[A(v)-U_{0}\left(x^{*}\right)\right]=\ln \frac{\beta v F(v) \ln 2}{\omega_{0}} .
$$

Equation (10) adds a modulation $F(v)$ to previous estimates $[10,12,14]$, which describe the enforced unbinding rate $(\propto v)$ by the Boltzmann factor, $\exp [-\beta \Delta A(\tau)]$.

Using (9), the derivative of (10) with respect to $v$ reads

$$
\frac{d \xi}{d v}=\frac{1}{\beta v F(v)}\left[1+\frac{v}{F(v)} \frac{d F(v)}{d v}\right],
$$

which can be solved numerically for any given force spectrum $F(v)$. From $\xi(v)$ and the given $F(v)$, a force profile $F(\langle x\rangle)$ as a function of the average reaction coordinate is readily obtained via $\langle x\rangle=\xi(v)-F(v) / k$ [cf. (6)]. From (2) and (5), and neglecting the anharmonicity of $V_{\text {pull }}$, this reconstructed force profile is identified as a convolution of the true profile, $F(x)=\partial_{x} U_{0}(x)$, with a Gaussian function of half-width $\sigma:=1 /(\beta k)^{1 / 2}$.

In the "soft spring regime" $\left(\tau>x^{*} / v\right), U\left(x^{*}\right)$ is already raised before $\tau$, and neither $x^{*}$ nor $U\left(x^{*}, \tau\right)$ are generally constant. Thus, for $\tau>x^{*} / v$, and using $Z_{b}$ as defined in (2), the time derivative in (8) reads

$$
\partial_{t} \Delta A(v)=k v \Delta x(v)+\left[k\left(\xi-x^{*}\right)-\partial_{x} U_{0}\left(x^{*}\right)\right] \partial_{t} x^{*},
$$

where $\Delta x:=x^{*}-\langle x\rangle$. At the maximum $U\left(x^{*}\right)$, one 
obtains

$$
\partial_{x} U\left(x^{*}\right)=\partial_{x} U_{0}\left(x^{*}\right)-F(v)+k \Delta x(v)=0 .
$$

By using (6), the second term in (12) vanishes, and (8) reads

$$
\beta\left[A(v)-U_{0}\left(x^{*}\right)\right]-\frac{k \beta}{2}\left[\Delta x-\frac{F(v)}{k}\right]^{2}=\ln \frac{\beta k v \Delta x}{\omega_{0}} .
$$

Comparison with (10) reveals a different modulation for the Boltzmann factor, which here results from the shift of $x^{*}$ due to $V_{\text {pull }}$ and a corresponding decrease of $\Delta x$.

In analogy to the above treatment of the stiff spring regime, the derivative of (14) with respect to $v$ relates $F(v)$ to a spatial coordinate: there to $\xi$, here to $\Delta x(v)$,

$$
\beta \Delta x(v)\left[k \frac{d\langle x\rangle}{d v}+\frac{d F(v)}{d v}\right]=\frac{1}{v}+\frac{1}{\Delta x} \frac{d \Delta x}{d v},
$$

where (13) and (9) were used.

To obtain a force profile $F(x)$ from (15), $\langle x\rangle$ remains to be specified as a function of $F(v)$, e.g., from stiff spring experiments. Without such data, the bound state may be assumed to be harmonic, i.e., $\langle x\rangle(v)=F(v) / k_{0}$ with $k_{0}:=\left.\partial_{x}^{2} U_{0}(x)\right|_{x=0}$. For this case, (11) can be solved,

$$
\Delta x(v)=\left[v \eta \beta \int_{v}^{\infty} d v^{\prime} \frac{1}{v^{\prime}} \frac{d F\left(v^{\prime}\right)}{d v^{\prime}}\right]^{-1},
$$

with $\eta:=1+k / k_{0}$. The integral in (16) converges if $F(v)$ is bounded from above by a polynomial in $\ln v$. If the curvature of $F(v)$ at a logarithmic velocity scale is small, (16) reduces to

$$
\Delta x(v)=\left[\eta \beta v \frac{d F(v)}{d v}\right]^{-1},
$$

which for $k \rightarrow 0$ recovers the TLS result used in [2].

Equations (11) and (16) relate any given force spectrum to the underlying force profile and are the main result of this Letter.

To explore accuracy and range of applicability of this theory we first study the forward transformation and, subsequently, the reconstruction. To that aim we considered the sample force profile shown in Fig. 2(a). From this profile we computed "exact" force spectra by numerical solution of (3) and (4) [Fig. 2(b), symbols] and compared these with the analytical results [19] for the stiff (10) and soft spring regimes (14) [Fig. 2(b), solid lines].

For $k>0.01 \mathrm{~N} / \mathrm{m}$ the spectra exhibit increasingly pronounced plateaus, which can be related to maxima in the force profile. Each of these plateaus ranges up to a critical velocity, at which the maximum of $F(v, t)$ exceeds the force defined by the preceding force maximum. Accordingly, a low maximum succeeding a larger one in the force profile [e.g., the third maximum in Fig. 2(a)] leaves no fingerprint in the force spectrum and, therefore, cannot be reconstructed. This behavior is well described by the stiff spring approximation.

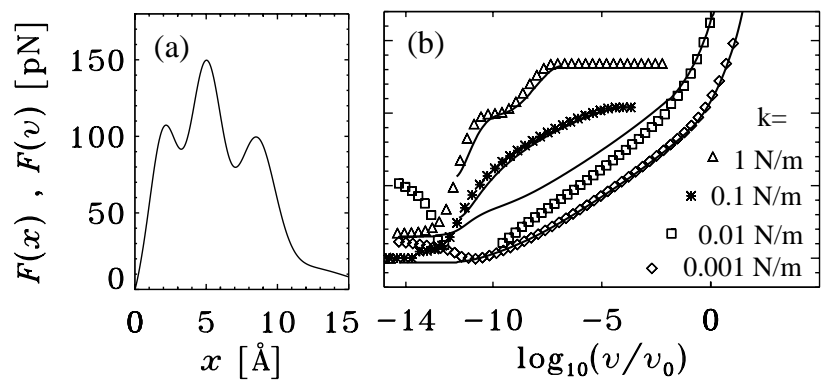

FIG. 2. Forward transformation: (a) sample force profile; (b) force spectra computed numerically (symbols) and analytically with (10) [upper two curves] and (14) [lower two curves] for different spring constants $k$. For clarity, the spectrum with $k=0.01 \mathrm{~N} / \mathrm{m}$ has been shifted upwards by $22 \mathrm{pN}$.

For small $k<0.01 \mathrm{~N} / \mathrm{m}$ the force spectrum becomes convex and is well described by the soft spring approximation, except for a small gap around $k=0.01 \mathrm{~N} / \mathrm{m}$, where both approximations perform poorly below $100 \mathrm{pN}$ due to the large value of the respective critical force, $F_{c} \approx 80 \mathrm{pN}$. Thus, for our example, the two approximations cover nearly the whole range of spring constants.

Second, to illustrate the primary application of the theory, we reconstructed a force profile from force spectra. Two of the numerical force spectra shown in Fig. 2(b) were used, namely, $k=10^{-3} \mathrm{~N} / \mathrm{m}$, which is a value typical for BFP measurements, and $k=1.0 \mathrm{~N} / \mathrm{m}$, which is likely to be accessible to AFM experiments in the near future. From each of the two spectra, 50 data points were taken as hypothetical experimental data. To mimic experimental error, a random scatter of $10 \%$ was added. Figure 3(a) displays the resulting stiff and soft spring data.

Figure 3(b) shows, together with the reference force profile (solid line), the reconstruction from the stiff spring $(\triangle)$ and from the soft spring data $(\diamond)$. For the former, (11) was discretized and solved recursively; for comparison, the convolution of the force profile with a Gaussian function of half-width $1 /(\beta k)^{1 / 2}$ is shown (dashed line). For the latter reconstruction, the discrete version of (16) was solved, where for each data point separate values for $k_{0}$ were taken from the stiff spring reconstruction, which were also used to convert $\Delta x$ to a reaction coordinate, $x=\Delta x-F / k_{0}$. "Experimental" data were preprocessed by smoothing with a Gaussian function. The activation barrier was chosen such that the energy landscape obtained from the force profile (inset) is smooth at the transition from the stiff $(\triangle)$ to the soft $(\diamond)$ spring regime.

Essential features, such as the heights and positions of the force maxima, are well reproduced. Furthermore, our scheme proves to be robust with respect to experimental scatter of yielding forces. Note that the maxima resolved resemble in size those attributed to the rupture of single hydrogen bonds (cf. Ref. [9]).

Finally, we have attempted to reconstruct in a similar manner the force profile and energy landscape [Fig. 3(d) and inset] from a recent single molecule BFP biotin/avidin 

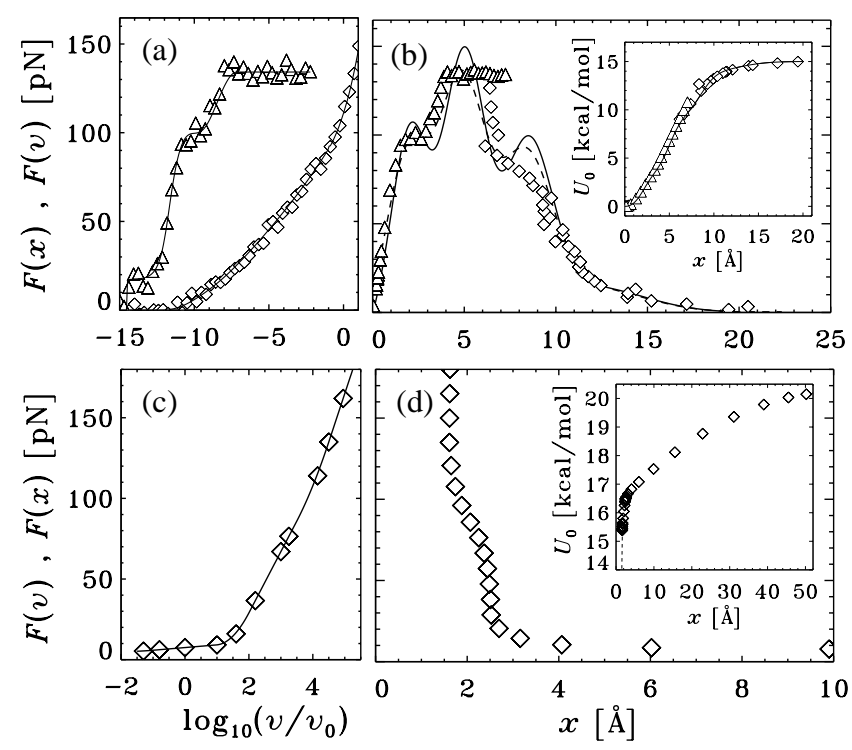

FIG. 3. Reconstruction of force profiles [(b,d), symbols] and energy landscapes (insets) from simulated [(a), symbols] and real [(c), symbols] experimental data. Simulated experimental data have been derived from the reference force profile shown in (b) (solid line) for stiff $(\triangle)$ and soft $(\diamond)$ springs. Single molecule biotin/avidin force spectra (c) were taken with permission from Ref. [2]. In (b) the convolution of the reference force profile with a Gaussian of half-width $\sigma$ is shown for comparison (dashed line). In (d) part of the energy landscape could not be reconstructed and is sketched as the dashed line.

force spectrum [2] [Fig. 3(c)]. The positions of the steps in the latter force profile agree well with the positions of the barriers proposed in Ref. [2]. Particularly, the surprisingly long range interaction discussed in Ref. [2] could be quantified. By construction, the derived energy landscape approximates the convex hull of the true one, which may contain wells.

We have developed a theory that relates single molecule dynamic force probe spectra to the free energy landscape that governs the molecular response to mechanical stress. This theory enables one to derive force profiles with high spatial resolution from measured force spectra, since our transformation from the space domain into the velocity domain circumvents experimental spatial resolution limits. In particular, soft and stiff spring experiments (e.g., BFP or optical tweezers versus AFM) are expected to provide complementary information and, therefore, should be combined. Our results suggest that force probe experiments covering a large range of loading rates should allow one to characterize in detail molecular pathways, e.g., of ligandreceptor unbinding, protein unfolding, polymer stretching, or molecular adhesion, with a spatial resolution at the level of single hydrogen bonds.
We thank E. Evans for providing his avidin/biotin data, and $\mathrm{H}$. Wagner for valuable suggestions. This work was supported by the DFG, Grant No. GR 1590/1-2.

[1] G. U. Lee, D. A. Kidwell, and R. J. Colton, Langmuir 10, 354 (1994); E.-L. Florin, V. T. Moy, and H. E. Gaub, Science 264, 415 (1994).

[2] R. Merkel et al., Nature (London) 397, 50 (1999).

[3] P. Hinterdorfer et al., Proc. Natl. Acad. Sci. U.S.A. 93, 3477 (1996); R. Ros et al., Proc. Natl. Acad. Sci. U.S.A. 95, 7402 (1998); D. A. Simson et al., Phys. Rev. Lett. 83, 652 (1999).

[4] M. Rief et al., Science 276, 1109 (1997).

[5] L. Tskhovrebova et al., Nature (London) 387, 308 (1997); A. F. Oberhauser et al., Nature (London) 393, 181 (1998).

[6] G. U. Lee, L. A. Chrisey, and R. J. Colton, Science 266, 771 (1994); T. Strunz et al., Proc. Natl. Acad. Sci. U.S.A. 96, 11277 (1999).

[7] M. Rief et al., Science 275, 1295 (1997).

[8] M. Rief, J. M. Fernandez, and H. E. Gaub, Phys. Rev. Lett. 81, 4764 (1998); P. E. Marszalek et al., Nature (London) 396, 661 (1998).

[9] H. Grubmüller, B. Heymann, and P. Tavan, Science 271, 997 (1996).

[10] S. Izrailev et al., Biophys. J. 72, 1568 (1997).

[11] A. Krammer et al., Proc. Natl. Acad. Sci. U.S.A. 96, 1351 (1999); H. Lu and K. Schulten, Proteins 35, 453 (1999); P. Marszalek et al., Nature (London) 402, 100 (1999).

[12] B. Heymann and H. Grubmüller, Chem. Phys. Lett. 303, 1 (1999); 305, 202 (1999); 307, 425 (1999).

[13] G. I. Bell, Science 200, 618 (1978); J. Shillcock and U. Seifert, Phys. Rev. E 57, 7301 (1998).

[14] E. Evans and K. Ritchie, Biophys. J. 72, 1541 (1997).

[15] M. Rief et al., Biophys. J. 75, 3008 (1998).

[16] E. Evans and K. Ritchie, Biophys. J. 76, 2439 (1999).

[17] For the semiharmonic potential at hand, a correction, $-\left(1 / Z_{b}\right) \int_{v t}^{x^{*}} d x k(x-v t) \exp \left[-\beta U_{0}(x)\right]$, can be neglected if $F(v, \tau)$ is larger than a critical force, $F_{c}:=$ $k /\left[\beta^{2}\left(k^{2}+k_{0}^{2}\right)\right]^{1 / 4}$. Here, $k_{0}=\partial_{x}^{2} U_{0}(x)$ at $x=0$ describes the localization of the bound state. For $k \gg k_{0}$ (stiff spring) the critical force approaches $F_{c} \approx(k / \beta)^{1 / 2}$; for $k \ll k_{0}$ (soft spring), $F_{c} \approx k /\left(\beta k_{0}\right)^{1 / 2}$ vanishes for $k \rightarrow 0$.

[18] Like $\overline{\Delta A}(t)$, also $P_{v}(t)$ is valid only within the yielding period. Closer inspection of $P_{v}(t)$ reveals, however, that, for forces $\partial_{t} A(t) / v$ of the order of or larger than the critical force $F_{c}, P_{v}(t)$ is a steeply decreasing sigmoidal function that approaches unity $(t<\tau)$ and zero $(t>\tau)$ already within the yielding period. In this case $P_{v}(t)$ is a good approximation to the exact solution of (3) for all $t \geq 0$.

[19] Individual yielding forces $F_{i}$ were taken from the sample profile. The respective coordinates $x_{i}$ defined yielding positions $\xi_{i}$, which served to numerically compute free energies $A_{i}$ via (2). For these values, the required $v_{i}$ were computed from (10) and (14), respectively. 